\title{
Suaeda japonica Makino Attenuates Lipopolysaccharide- Induced Neuro-Inflammatory Responses in BV-2 Microglia via NF-kappa B Signaling
}

\author{
Hyun Kang ${ }^{1,3 \dagger}$, Sushruta Koppula ${ }^{1 \dagger}$, Hoi- Ki Kim ${ }^{2}$ and Tae-Kyu Park ${ }^{1^{*}}$ \\ ${ }^{1}$ Department of Biotechnology, Research Institute for Biomedical \& Health Science, College of Biomedical and Health Science, \\ Konkuk University, Chungju, 380-701, ${ }^{2}$ Fanipin Korea Co, Ltd, Ochang 208-211, ${ }^{3}$ KuGen Healthcare Institute, Chungju, 380- \\ 150, Republic of Korea
}

*For correspondence: Email: tkpark@kku.ac.kr; Tel: 82-43-840-3870; Fax: 82-43-852-3616

Received: 18 April 2013

Revised accepted: 23 May 2013

\begin{abstract}
Purpose: To evaluate in vitro anti-oxidant and anti-neuro-inflammatory activities of Suaeda japonica extract (SJE) in lipopolysaccharide (LPS)-stimulated BV-2 microglial cells.

Methods: 1, 1-diphenyl-2-picryl-hydrazyl (DPPH) radical scavenging assay was used to study the antioxidant effects. 3-(4, 5-dimethylthiazol-2-yl)-2, 5- diphenyl-tetrazolium bromide (MTT) assay was used to study cell viabilities. LPS-stimulated BV- microglial cells were used to study the gene expression and production of inflammatory mediators determined by Western blot analysis.

Results: SJE significantly inhibited the DPPH generated free radicals, and suppressed LPS-induced expression of inducible nitric oxide synthase (iNOS) and production of nitric oxide (NO) in a concentration-dependent manner. It decreased LPS-induced expression of some inflammatory mediators and pro-inflammatory cytokines (cyclooxygenase-2 and interleukin (IL)-6). This suppression of inflammatory mediators was nuclear factor kappa-B (NF-kB)-dependent.

Conclusion: Our findings imply that SJE may be a potential therapeutic agent in regulating microgliamediated neuroinflammatory responses observed in several neurodegenerative diseases.
\end{abstract}

Keywords: Suaeda japonica, Antioxidant activity, Anti-inflammatory activity, Microglial Cells, iNOS, IL6.

Tropical Journal of Pharmaceutical Research is indexed by Science Citation Index (SciSearch), Scopus, International Pharmaceutical Abstract, Chemical Abstracts, Embase, Index Copernicus, EBSCO, African Index Medicus, JournalSeek, Journal Citation Reports/Science Edition, Directory of Open Access Journals (DOAJ), African Journal Online, Bioline International, Open-J-Gate and Pharmacy Abstracts

\section{INTRODUCTION}

Suaeda japonica Makino (S. japonica), from the family Chenopodiaceae, is an annual succulent herb and one of the most salt-tolerant plant species seen abundantly on muddy seashores along the western coast of Korea, Europe, Japan and Iran [1]. Suaeda species have been used as a seasoning vegetable, salad and fermented food and also as a folk medicine for treating obesity and diabetes [2]. Recently, S. japonica has been used as functional food and as well as medicinal plant due to its beneficial effects such as a-glusidase inhibition, antioxidant and antidiabetic activities [3, 4]. However, there are no studies on its possible beneficial effects on neuro-inflammatory diseases.

Neuro-inflammation mediated by microglial activation appears to play an essential role in the pathogenesis of various neuro-degenerative diseases such as Alzheimer's disease, Parkinson's disease and multiple sclerosis [5]. Microglia under activated conditions releases several inflammatory cytokines and toxic free 
radicals which may participate in the neurodegenerative process. Previous reports strongly demonstrate that agents that suppress elevation of inflammatory mediators in activated microglia can attenuate the severity of neuro-degenerative diseases [6].

It is well documented that the pathophysiological condition in inflammatory disorders is initiated by a complex processes triggered by microbial endotoxins like LPS, which can directly activate microglia, thus triggering the production of inflammatory mediators, such as NO, COX-2, pro-inflammatory cytokines and leukotrienes [7]. Therefore, LPS-induced inflammatory mediators in vitro can be regarded as one of the essential tools for evaluating the deleterious consequences of excessive activation of microglia. Recent studies have shown that antioxidant and anti-inflammatory agents may suppress microglial activation and thus protect neuronal cell death [8,9]. In this study we evaluated the in vitro antioxidant potential and anti-neuroinflammatory properties of SJE in LPSstimulated BV-2 microglial cells.

\section{EXPERIMENTAL}

\section{Preparation of S. japonica extract}

Whole plant of $S$. japonica was collected from the western coast region of South Korea in the month of April, 2011. The plant was authenticated by a taxonomist at Konkuk University, South Korea and a voucher specimen (SJ-KU2013) was stored in department of Plant Biotechnology herbarium, Konkuk University for future reference. To obtain the $S$. japonica extract, the dried plant material was ground in a blender and defatted three times with three volumes of ethanol. The residue was extracted with absolute ethanol at 1:10 ratio $(w / v)$ for $2 \mathrm{~h}$ in a heating mantle at $70-80{ }^{\circ} \mathrm{C}$, and the supernatant was filtered and concentrated in a vacuum evaporator system at $50^{\circ} \mathrm{C}$. For further fractionation, the extract $(1000 \mathrm{mg})$ was partitioned into hexane, chloroform and ethyl acetate (EA) fractions to yield 235, 35 and 356 $\mathrm{mg}$, respectively. The active EA fraction of $S$. japonica (SJE) was re-dissolved in distilled water to evaluate its anti-neuroinflammatory and antioxidant activities.

\section{DPPH radical scavenging activity}

The antioxidant activity of the SJE was determined using the stable radical 2, 2-diphenyl1-picrylhydrazyl (DPPH, Sigma-Aldrich, St. Louis, MO, USA). The radical scavenging capacity was evaluated by employing a reaction mixture constituted by aliquots of the SJE extract and a DPPH methanolic solution as described previously [10]. Briefly, a sample solution of $60 \mu \mathrm{l}$ of each OFP-EA extract, was added to $60 \mu \mathrm{l}$ of DPPH $(60 \mu \mathrm{M})$ in methanol. After mixing vigorously for $10 \mathrm{~s}$, the mixture was transferred into a $100 \mu$ Teflon capillary tube and the scavenging activity of each sample on DPPH radical was measured using a JES-FA ESR spectrometer (Jeol Ltd., Tokyo, Japan). A spin adduct was measured on an ESR spectrometer exactly after $2 \mathrm{~min}$. Experimental conditions were as follows: central field, 3,475 G; modulation frequency, $100 \mathrm{kHz}$; modulation amplitude, $2 \mathrm{G}$; microwave power, $5 \mathrm{~mW}$; gain, $6.3 \times 10^{5}$, and temperature, $298^{\circ} \mathrm{K}$.

\section{Cell cultures and viabilities}

BV-2 microglia cells were cultured at $37^{\circ} \mathrm{C}$ in 5 $\% \mathrm{CO}_{2}$ in DMEM (Invitrogen, Carlsbad, CA, USA) supplemented with $5 \%$ Foetal Bovine Serum (FBS, Hyclone, Logan, UT, USA) and antibiotics (Invitrogen). In all experiments, cells were pre-treated with the indicated concentrations of SJE for $1 \mathrm{~h}$ before the addition of LPS ( $1 \mu \mathrm{g} / \mathrm{ml}$, Sigma-Aldrich, St. Louis, MO, USA) in serum free DMEM. An equal volume of sterile water was added to all control treatments. Cell viability was determined by 3-(4, 5dimethylthiazol-2-yl)-2,5-diphenyltetrazolium

bromide (MTT) assay as described previously [11]. Cells were incubated with various concentrations of SJE for $24 \mathrm{~h}$ followed by MTT for $4 \mathrm{~h}$, and then $100 \mu \mathrm{l}$ of isopropanol (in $0.04 \mathrm{~N}$ hydrochloric acid) was added to dissolve the formazan crystals. The absorbance was read at $570 \mathrm{~nm}$ using the Anthos 2010 spectrophotometer (Salzburg, Austria). Cell viability was calculated as relative absorbance compared to control.

\section{Nitric oxide assay}

The amount of stable nitrite, the end product of NO generation, by activated microglia was determined by a colorimetric assay as previously described [12]. Briefly, $50 \mu \mathrm{l}$ of culture supernatant was mixed with an equal volume of Griess reagent and incubated at room temperature for $10 \mathrm{~min}$. The absorbance at 540 $\mathrm{nm}$ was read using a PowerWavex Microplate Scanning spectrophotometer (Bio-Tek Instrument, Winooski, VT, USA). Nitrite concentration was determined by extrapolation from a sodium nitrite standard curve. 


\section{Nuclear protein extraction and Western blot analysis}

Cells were washed in cold PBS three times and lysed in a buffer containing $50 \mathrm{mM}$ Tris- $\mathrm{HCl}, \mathrm{pH}$ 7.4, $1 \%(\mathrm{v} / \mathrm{v})$ Tergitol- type NP-40 (NP-40), $0.25 \%$ sodium deoxycholate, $150 \mathrm{mM} \mathrm{NaCl}, 1$ $\mathrm{mM}$ EDTA, $25 \mathrm{mM} \mathrm{NaF}, 2 \mathrm{mM}$ Sodium orthovanadate $\left(\mathrm{Na}_{3} \mathrm{VO}_{4}\right)$ and protease inhibitor cocktail (Complete Mini ${ }^{\mathrm{TM}}$, Roche, Mannheim, Germany) at $4{ }^{\circ} \mathrm{C}$. The lysate was clarified by centrifugation at $10,000 \mathrm{~g}$ for $20 \mathrm{~min}$ at $4{ }^{\circ} \mathrm{C}$ to remove insoluble components. Cell lysates were normalized for protein content using bicinchoninic acid (BCA) reagent (Pierce, Rockford, IL, USA). Equal amounts of protein were loaded onto $10 \%$ poly acryl amide gel electrophoresis (PAGE) and separated by standard sodium dodecyl sulphate (SDS)-PAGE procedure. Proteins were transferred to an NC membrane (S\&S, Dassel, Germany) and blocked with $5 \%$ non-fat dry milk in TBS. To detect protein expression, the blots were probed with the specific antibodies followed by the secondary antibodies coupled to horseradish peroxidase (Bio-Rad, Herculus, CA, USA) with $\beta$-actin as internal control. The immunoreactive proteins on the membrane were detected by chemiluminescence using the West-Save substrate (Lab-Frontier, Seoul, Korea) on X-ray film. The antibodies against inducible nitric oxide synthase (iNOS), cyclooxegenase (COX) -1 , COX-2, nuclear factor kappa-B (NF-kB), I kappa B-alpha (IKB- $\alpha$ ) and $\beta$-actin were purchased from Cell Signaling Technology Inc (Beverly, MA, USA).

\section{IL-6 assay}

BV-2 microglia cells $\left(1 \times 10^{5}\right.$ cells/well) were cultured on 96 well plates and treated with SJE at indicated dosages with or without LPS $(1 \mu \mathrm{g} / \mathrm{ml})$. At $4 \mathrm{hr}$ of post LPS treatment, the cells were collected and the supernatants were subjected to assay of IL-6 contents using a murine IL-6 ELISA kit from BD Biosciences (San Jose, CA, USA) according to the manufacturer's instruction.

\section{Statistical analysis}

All data are represented as the mean \pm S.E.M of at least three independent experiments. Statistical analyses were performed using SAS statistical software (SAS Institute, Cray, NC, USA) using one-way analysis of variance, followed by Dunnett's multiple range tests. $P<$ 0.05 was considered statistically significant.

\section{RESULTS}

\section{Effect of SJE extract on DPPH radical scavenging activity}

As shown in Fig. 1A, SJE exhibited significant DPPH radical scavenging activity in a dosedependent manner showing a maximum effect at $100 \mu \mathrm{g} / \mathrm{ml}$ of concentration $(p<0.001)$. The ESR spectroscopy data at 10,50 and $100 \mu \mathrm{g} / \mathrm{ml}$ are shown in Fig 1B.
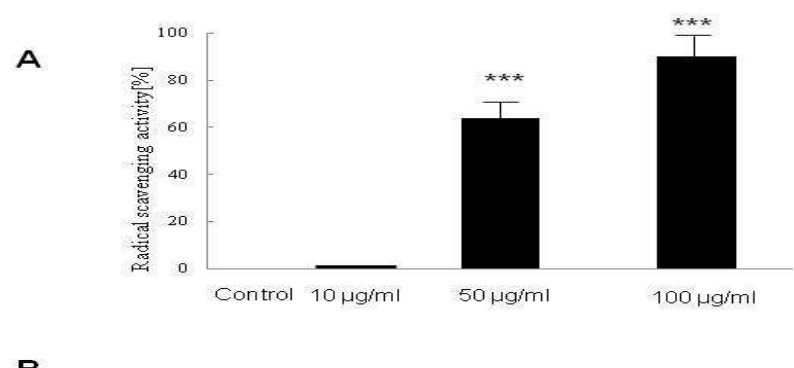

B

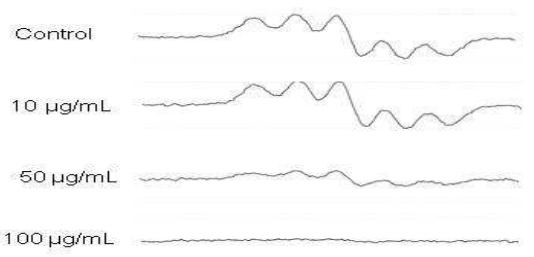

Fig 1: Effect of SJE on DPPH radical scavenging activity. The capacity to scavenge DPPH free radical by different concentrations of SJE (A) and ESR spectra (B) was measured. BV-2 cells were treated with or without SJE at the various concentrations (10, 50 and $100 \mu \mathrm{g} / \mathrm{ml})$. The scavenging activity of each sample on DPPH radical was measured using a JESFA ESR spectrometer. A spin adduct was measured on an ESR spectrometer exactly 2 min later. Data are presented as the mean \pm S.E.M. $(n=3)$ for three independent experiments. ${ }^{* * *} p<0.001$, when compared with control group by one-way analysis of variance, followed by Dunnett's multiple range tests. SJE = Suaeda japonica ethyl acetate extract.

\section{Effect of SJE on LPS-induced NO production}

NO production by LPS-activated cells significantly inhibited by SJE in a concentrationdependant manner (Fig 2).

SJE treatment did not exhibit any significant cytotoxicity in BV-2 microglial cells even at a high concentration $(200 \mu \mathrm{g} / \mathrm{ml})$ up to $24 \mathrm{~h}$ of incubation, and in all cases the viability was found above $93 \%$ by MTT assay (Fig 3).

\section{Effect of SJE on LPS-induced expression of iNOS and COX levels}

SJE exhibited a broad spectrum of inhibitory effect on the expression of pro-inflammatory 
mediators and reduced the LPS-stimulated increase of protein expression such as iNOS and inducible COX-2 in a concentration-dependent manner. However, constitutive COX-1 protein expressional levels were uninterrupted (Fig 4).

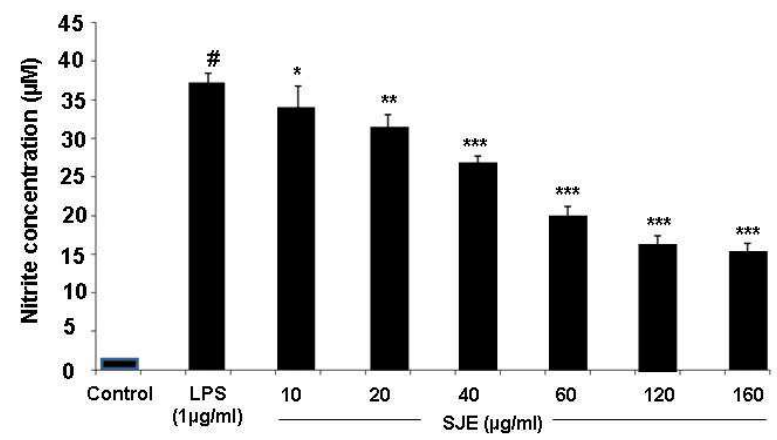

Fig 2: Effect of SJE on NO Production in LPSstimulated BV-2 microglial cells. BV-2 cells were treated with SJE at various concentrations (10, 20, 40, $80,120$ and $160 \mu \mathrm{g} / \mathrm{ml})$ with or without LPS $(1 \mu \mathrm{g} / \mathrm{ml})$ for $4 \mathrm{hr}$. The nitrite in the culture supernatant was evaluated using Griess reagent. Data are presented as the mean \pm SEM $(n=3)$ for three independent experiments. ${ }^{\#} p<0.001$, when compared with control group. ${ }^{*} p<0.05,{ }^{* *} p<0.01$ and ${ }^{* * *} p<0.001$, when compared with LPS alone treated group by one-way analysis of variance, followed by Dunnett's multiple range tests. SJE = Suaeda japonica ethyl acetate extract.

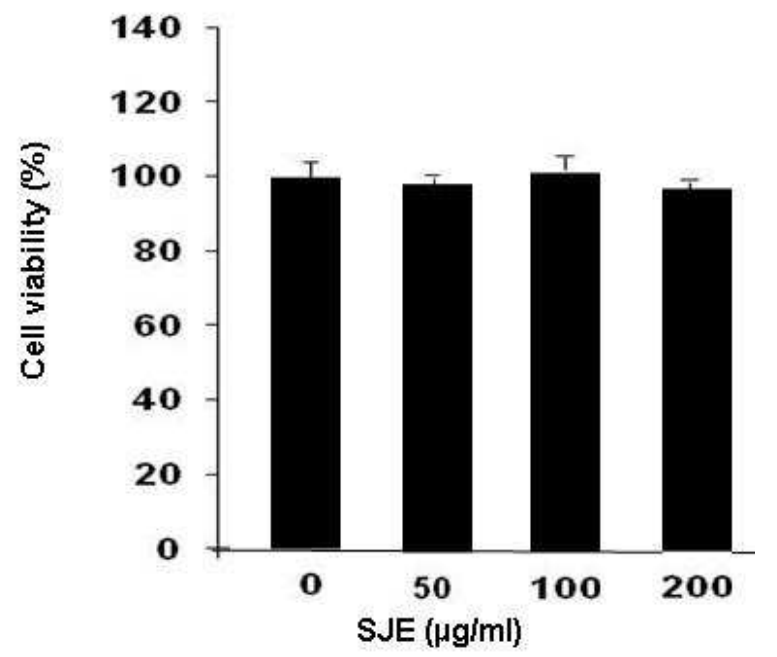

Fig 3: Effect of SJE on the viability of BV-2 microglial cells. Data are presented as the mean $\pm \operatorname{SEM}(n=3)$ for three independent experiments. SJE $=$ Suaeda japonica ethyl acetate extract.

\section{Effect of SJE on NF-kB levels}

SJE inhibited the LPS-induced phosphorylation and degradation of IKB- $\alpha$, and nuclear translocation of p65 NF-KB in a concentrationdependent manner (Fig. 5).
Effect of SJE on IL-6 production in LPSstimulated $B V-2$ cells

LPS stimulation increased the IL-6 expression in BV- cells. However, pretreatment with SJE decreased the LPS-induced IL-6 levels in BV-2 microglia (Fig. 6).

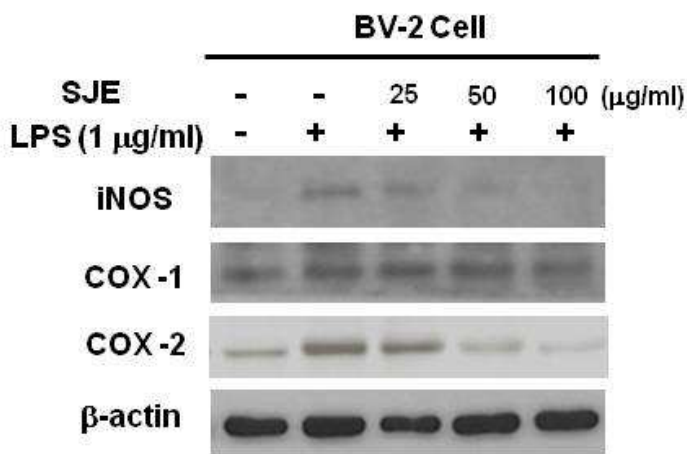

Fig 4: Effect of SJE on iNOS, COX-1 and COX-2 protein expressional levels in LPS-stimulated BV-2 microglial cells. The expression levels of iNOS, COX-1 and COX-2 production in the LPS-stimulated BV-2 cells by various concentrations $(25,50$ and $100 \mu \mathrm{g} / \mathrm{ml})$ of SJE was monitored by immunoblot analyses with the specific antibodies against iNOS, COX-1 and COX-2. The internal control used was $\beta$-actin. SJE = Suaeda japonica ethyl acetate extract.

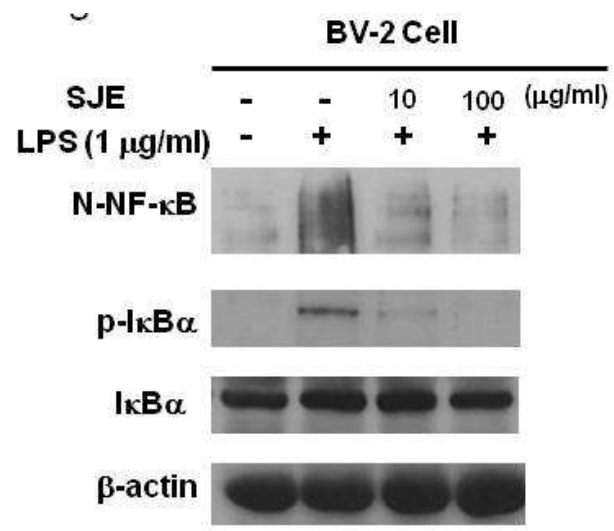

Fig 5: Effect of SJE on NF-kB activity in LPSstimulated BV2 microglia. Data are presented as the mean \pm SEM $(n=3)$ for three independent experiments. SJE = Suaeda japonica ethyl acetate extract.

\section{DISCUSSION}

It is well documented that microglia produces NO in response to pro-inflammatory stimuli [13]. The NO produced by the induction of iNOS will lead to increased inflammatory reaction. Therefore, agents that decrease NO production and iNOS have appreciable therapeutic effect in the treatment of several neuro-inflammatory diseases [13]. Our results clearly showed that SJE attenuated LPS-induced iNOS expression and downstream NO production. 
Earlier literature revealed that increased expression of the COX-2 plays an important role in the inflammatory process and neurodegenerative diseases [14]. LPS strongly activates microglia and induces COX-2.

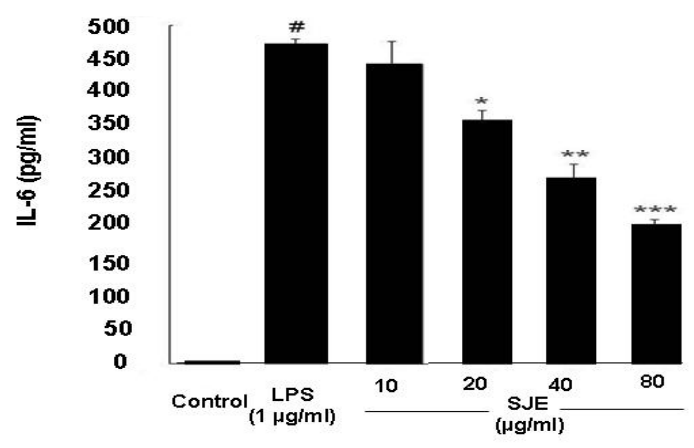

Fig 6: Effect of SJE on pro-inflammatory cytokine IL-6 expression in LPS-stimulated BV-2 cells. Data are presented as the mean \pm SEM $(n=3)$ for three independent experiments. ${ }^{\#} p<0.001$, when compared with control group. ${ }^{*} p<0.05,{ }^{* *} p<0.01,{ }^{* * *} p<0.001$, compared with group treated with LPS only; comparison made by one-way analysis of variance, followed by Dunnett's multiple range tests. SJE= Suaeda japonica ethyl acetate extract.

Several reports indicate that iNOS and COX-2 are induced in various types of central nervous injuries and diseases [14, 15]. Therefore attenuation of the induction of iNOS and COX-2 in activated microglia could inhibit neuroinflammation. Although our results point to a potential role of SJE in inhibiting iNOS and COX2 production, the possibility remains that SJE may further suppress other microglia-derived pro-inflammatory factors upon LPS treatment in BV-2 cells. Pro-inflammatory cytokines such as TNF- $\alpha$, IL- $1 \beta$ and IL- 6 cause potent activation of iNOS gene expression in rodent glial cells and muscle cells [15]. The likelihood of the involvement of SJE in attenuating such factors is supported by our observations that proinflammatory mediator IL-6 produced by LPS treatment was suppressed by SJE in BV-2 microglial cells indicating that SJE may be an effective anti-neuro-inflammatory agent.

NF-kB, a mammalian transcription factor, activated by LPS, is known to control the expression of cell survival genes as well as proinflammatory enzymes and cytokines [16]. Our result showed that SJE inhibited the LPSinduced phosphorylation/degradation of IKBa and translocation of NF-kB/p65 sub-unit in a concentration-dependent manner. We can conclude that NF-KB is a major target of SJE. However, the exact molecular target of SJE on NF-kB activation remains to be elucidated.
$\mathrm{DPPH}$ radical assay is one of the widely used methods for screening the free radical scavenging activities of several anti-oxidants in a relatively short period of time []. Microglia under activated conditions releases toxic free radicals which may participate in the neurodegenerative process. In the present study, SJE significantly scavenged the DPPH free radicals supporting the anti-neuroinflammatory effects in LPSstimulated BV-2 cells. Earlier reports indicate that Suaeda species possess active constituents such as triterpenoids and anthocyanins such as betacyanin, dioxyisoflavones, loliolide, uridine and dehydrovomifoliol $[17,18]$. Triterpenoids and anthocyanins including betacyanin are known to possess antioxidant properties [19, 20].

\section{CONCLUSION}

The present study reveals for the first time that SJE inhibits inflammatory responses through the inhibition of NF-kB signaling in LPS-stimulated microglia. The antioxidant potential of SJE might be responsible for the observed effects

\section{CONFLICT OF INTEREST}

The authors declare that no conflict of interest as far as this work is concerned.

\section{ACKNOWLEDGEMENT}

This work was supported by Business for Cooperative R\&D (Grant no. C0034986) between Industry, Academy, and Research Institute-funded Korea Small and Medium Business Administration, 2012. 'The authors, HK and SK, contributed equally to this work.

\section{REFERENCES}

1. Flowers TJ, Troke $P$, Yeo AR. Mechanism of salt tolerance in halophytes. Annu. Rev. Plant Physiol 1977; 28: 89-121.

2. Benwahhoud $M$, Jouad $H$, Eddouks $M$, Lyoussi $B$. Hypoglycemic effect of Suaeda fruticosa in streptozotocin-induced diabetic rats. J Ethnopharmacol 2001; 76: 35-38.

3. Huang Z, Park SY, Feng L, Cho JY, Ham KS. Effects of a halophyte Suaeda japonica on Anti-Diabetic Activity in OLETF Rats (Symposium). International Symposium and Annual meeting 2011; 10: 416416. Available from: http://www.dbpia.co.kr/ Journal/ArticleDetail/1613331.

4. Kunkel G. Plants for Human Consumption. Koeltz Scientific Books, ISBN 3874292169, 1984.

5. Perry VH, Gordon S. Macrophages and microglia in the nervous system. Trends Neurosci 1988; 11: 273277.

6. Liu B, Hong JS. Role of microglia in inflammationmediated neurodegenerative diseases: mechanisms and strategies for therapeutic intervention. J Pharmacol Exp Ther 2003; 304: 1-7.

7. Xie $Q W$, Kashiwabara $Y$, Nathan C. Role of transcription 
factor NF-kappa B/Rel in induction of nitric oxide synthase J Biol Chem 1994; 269(7): 4705-4708.

8. Agullo G, Gamet-Payrastre L, Manenti S, Viala $C$ Rémésy C, Chap H, Payrastre B. Relationship between flavonoid structure and inhibition of phosphatidylinositol 3-kinase: a comparison with tyrosine kinase and protein kinase $C$ inhibition. Biochem Pharmacol 1997; 53: 2087-2094.

9. Araki E, Forster C, Dubinsky JM, Ross ME, ladecola C. Cyclooxygenase-2 inhibitor NS-398 protects neuronal cultures from lipopolysaccharide-induced neurotoxicity. Stroke 2001; 32: 2370-2375.

10. Sanches-Moreno C, Plaza L, Ancos B, Cano MP. Nutritional characterization of commercial trnaditional pasteurized tomato juices: carotenoids, vitamin and radical-scavenging activity. Food Chem 2006; 98: 749-756.

11. Tang YJ, Yang JS, Lin CF, Shyu WC, Tsuzuki M, Lu CC, Chen YF, Lai KC. Houttuynia cordata Thumb extract induces apoptosis through mitochondrialdependent pathway in HT-29 human colon adenocarcinoma cells. Oncol Rep 2009; 22: 1051. 1056

12. Green LC, Wagner DA, Glogowski J, Skipper PL, Wishnok JS, Tannenbaum SR. Analysis of nitrate, nitrite, and $\left[{ }^{15} \mathrm{~N}\right]$ nitrite in biological fluids. Anal Biochem. 1982; 126: 131-138.

13. Boucher JL, Moali C, Tenu JP. Nitric oxide biosynthesis, nitric oxide synthase inhibitors and arginase competition for L-arginine utilization. Cell Mol Life Sci 1999; 55:1015-1028.
14. Teismann $P$, Vila $M$, Choi DK, Tieu $K$, Wu DC, JacksonLewis V, Przedborski S. COX-2 and neurodegeneration in Parkinson's disease. Ann. N.Y. Acad. Sci 2003; 991: 272-277.

15. Hunot $S$, Hartmann $A$, Hirsh EC. The inflammatory response in the Parkinson brain. Clin Neurosci Res 2001; 1: 434-443.

16. Lee S, M, Yune TY, Kim SJ, Kim YC, Oh YJ, Markelonis GJ. Minocycline inhibits apoptotic cell death via attenuation of TNF-alpha expression following iNOS/NO induction by lipopolysaccharide in neuron/glia co-cultures. J Neurochem 2004; 91: $568-578$

17. Kim JS, Lee S, Son EM, Pan X, Kim UA, Lee GS, Seo $Y$, Lee BJ. Phytochemical constituents of Suaeda japonica Makino. Korean J Crop Sci 2005; 50: 208210.

18. Ghosh A, Misra S, Dutta AK, Choudhury A. Pentacyclic triterpenoids and sterols from seven species of mangrove. Phytochem 1985; 24: 1725-1727.

19. Subrahmanyam $C$, Rao KB, Rao CV, Rao BV. Chemical examination of the mangrove species Suaeda maritima and S. monica. Acta Cien Ind 1992; 18:78.

20. Hayakawa $K$, Agarie S. Physiological Roles of Betacyanin in a Halophyte, Suaeda japonica Makino. Plant Prod. Sci 2010; 13(4): 351-359. 